\title{
Aceptación por parte de estudiantes de la ETSID del curso de inglés, InGenio FCE Online Course \& Tester
}

Ana Gimeno Sanz

Grupo de Investigación CAMILLE, Departamento de Lingüística Aplicada, Universitat Politècnica de València agimeno@upvnet.upv.es

\begin{abstract}
The study described in this paper is based on the process carried out to validate the InGenio FCE Online Course \& Tester, developed by the CAMILLE research group at the Universitat Politècnica de València, as a suitable tool for learners wishing to take, and successfully pass, the Cambridge First Certificate in English examination. This validation process was carried out by means of a pre-course survey and a post-course survey in the form of two online questionnaires with a total of 146 learners of higherintermediate English enrolled in various technical degrees at the said University. There was sufficient evidence to corroborate its usefulness although a number of recommendations for improvements were revealed, which would have gone undiscovered without conducting the survey.
\end{abstract}

Keywords: Computer-Assisted Language Learning, Courseware validation, InGenio Online FCE Course and Tester.

\begin{abstract}
Resumen
El estudio que se describe en este artículo se basa en el proceso que se llevó a cabo para validar el curso online InGenio FCE Online Course \& Tester desarrollado por miembros del Grupo de Investigación CAMILLE de la Universitat Politècnica de València (UPV) como una herramienta eficaz para preparar a los estudiantes para realizar y aprobar el examen Cambridge First Certificate in English. El proceso de validación se llevó a cabo mediante la recogida de datos a través de dos encuestas de opinión; una administrada antes de que los estudiantes iniciaran el curso online y otro tras completarlo. La población encuestada ascendió a 146 estudiantes matriculados en varias de las titulaciones de grado de la UPV, con un nivel intermedio alto. A través del estudio se ha podido comprobar que el mencionado curso online cumplia sus objetivos aunque se detectaron algunas mejoras a realizar, que sin el estudio habrían pasado desapercibidas.
\end{abstract}

Palabras clave: Enseñanza de Lenguas Asistida por Ordenador, validación de cursos online, InGenio Online FCE Course and Tester. 


\section{Introducción}

Dada la escasez de materiales online para estudiantes de inglés para fines específicos (IFE) y el requisito de que los estudiantes de la Universitat Politècnica de València (UPV) hayan de acreditar un nivel de lengua extranjera equivalente al B2 del Marco común europeo de referencia para las lenguas (MCERL, Ministerio de Educación, Cultura y Deporte, 2002) para graduarse, algunos miembros del Grupo de Investigación CAMILLE de la UPV ${ }^{i}$ se embarcaron en un proyecto de desarrollo multimedia que daría como fruto la publicación del curso InGenio FCE Online Course \& Tester ${ }^{i i}$, con una triple finalidad: a) aprendizaje de inglés de nivel B2; b) preparación para realizar el examen del Cambridge First Certificate in English (FCE), y c) integrar contenido técnico relacionado con las materias impartidas en varias de las titulaciones de la Escuela Técnica Superior de Ingeniería del Diseño (ETSID). Además, los autores del curso han ido recogiendo datos mediante dos encuestas durante 3 cursos académicos consecutivos para conocer la percepción y la actitud de los estudiantes a la hora de estudiar inglés con un curso online. La primera de las encuestas tenía como finalidad recoger datos sobre el perfil de los estudiantes y sus expectativas de aprendizaje y, la segunda, recoger datos sobre su experiencia estudiando inglés con el curso online, sobre su grado de satisfacción y su percepción respecto a lo que habían mejorado. En las secciones que siguen se presentarán los resultados obtenidos haciendo especial hincapié en aquellos aspectos de mejora que se detectaron y las modificaciones que, en consecuencia, se han introducido en el curso. Para cerrar se concluirá con una serie de recomendaciones relacionadas con el desarrollo de cursos online destinados al aprendizaje de lenguas extranjeras.

\section{Objetivos}

Los objetivos del estudio que aquí se presenta se centraron en validar el desarrollo del curso InGenio FCE Online Course \& Tester como herramienta efectiva en tres aspectos fundamentales: a) para ayudar a los estudiantes a afianzar un nivel intermedio alto de inglés; b) para exponer a los estudiantes a temas y vocabulario en inglés relacionados con sus carreras universitarias, y c) para prepararlos para presentarse con éxito al examen oficial de la Universidad de Cambridge, First Certificate in English, que es equiparable al nivel B2 del MCERL, nivel que ha de acreditar todo estudiante de la UPV para graduarse.

\section{Desarrollo de la innovación}

\subsection{El curso InGenio FCE Online Course \& Tester}

El curso InGenio FCE Online Course \& Tester fue creado con la herramienta de autor del Sistema InGenio que se compone de cuatro módulos: a) una herramienta de autor para la creación y publicación de cursos de idiomas online, b) un entorno de aprendizaje, c) una herramienta para traducir a cualquier L1 (lenguas materna) la lengua vehicular de 
cualquiera de los cursos creados con la herramienta y, por último, d) un sistema de tutorización online para hacer un seguimiento exhaustivo del trabajo que realizan los estudiantes usando los cursos de InGenio.iii El curso se publicó en el año 2012 y se compone de dos módulos. Cada uno de éstos está dividido en dieciséis unidades didácticas de teoría y práctica. Ocho de estas corresponden a las actividades para afianzar conceptos teóricos y poner en práctica las cuatro destrezas lingüísticas (Course Units), mientras que las otras ocho simulan los ejercicios y las actividades propios del examen oficial de Cambridge para que los estudiantes puedan valorar si su nivel es equiparable al que se exige en dicho examen (Tester Units). Las unidades didácticas siguen el mismo patrón y están organizadas de la misma forma que el examen por lo que los contenidos se distribuyen en las siguientes secciones: comprensión escrita (Reading), expresión escrita (Writing), la lengua en uso (Use of Language), comprensión oral (Listening) y expresión oral (Speaking), y reúnen aproximadamente 50 actividades con un número variable de ítems. El tiempo de estudio asignado a cada módulo del curso es de 4,5 ECTS.

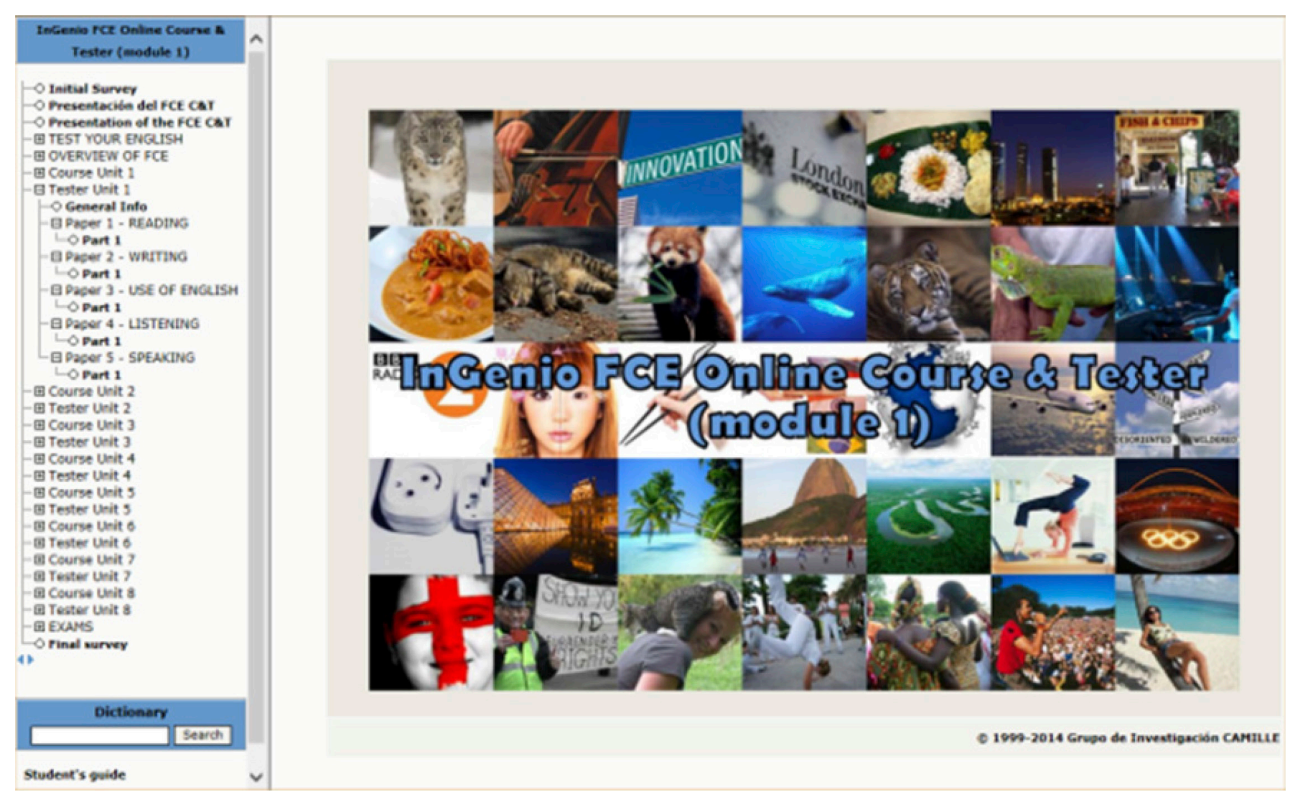

Fig. 1 Página de entrada. Module 1. InGenio FCE Online Course \& Tester

El curso InGenio FCE Online Course \& Tester es el primero que se ha publicado en el mundo con estas características.

\subsection{Método}

A lo largo de tres cursos académicos, se les administró a los estudiantes matriculados en el curso InGenio FCE Online Course \& Tester dos encuestas de opinión utilizando para ello Google Forms y habiéndolas integrado en el curso. La primera de ellas la realizaron antes de iniciar las actividades del curso y la segunda tras completarlo. Hubo un total de 146 estudiantes que realizó la primera encuesta pero sólo 66 de ellos completó el curso entero y la encuesta final, lo que supuso un $45,2 \%$ de los inscritos. Sus edades variaban entre los 18

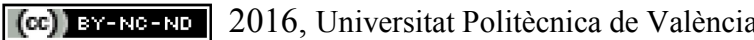

Congreso In-Red (2016) 
y los 23 años. Todos ellos estaban matriculados en titulaciones de grado de la ETSID. La encuesta inicial estaba compuesta de 5 apartados con un total de 66 preguntas basadas en una escala Likert de 5 puntos más 17 preguntas abiertas. El contenido era el siguiente:

1. Información personal y la percepción del estudiante respecto a su facilidad para aprender idiomas y cuán importante percibe el conocimiento de lenguas extranjeras para sus estudios y su futuro profesional (20 ítems).

2. El uso que hacen de las tecnologías de la información y de las comunicaciones tanto como herramientas de estudio como de ocio, y su actitud sobre el uso de la tecnología como herramienta educativa (21 ítems).

3. Información sobre sus expectativas ante un curso de estas características y su motivación para escoger esta asignatura y seguir aprendiendo inglés y las preferencias que tienen respecto a su aprendizaje (12 ítems).

4. Información sobre los estilos de aprendizaje y los métodos que prefieren (6 ítems).

5. Su grado de conocimiento respecto al MCERL y el examen del FCE (7 ítems).

El cuestionario final también estaba dividido en 5 apartados, pero con un total de 80 preguntas a contestar sobre una escala de Likert de 7 puntos, además de 3 preguntas abiertas. Las preguntas giraban en torno a los siguientes temas:

1. Características generales del curso para comprobar el nivel de satisfacción sobre la navegación, la interfaz gráfica, sobre lo intuitivo que resulta, la relevancia de los contenidos, adecuación del nivel lingüístico, equilibrio entre teoría y práctica, adecuación del tiempo asignado para su realización, grado de autonomía, satisfacción con el apoyo del tutor (profesor), calidad de los diversos media, etc. (22 ítems).

2. Información sobre los contenidos: calidad de las instrucciones, utilidad de los ejercicios para practicar las cuatro destrezas lingüísticas (comprensión y expresión oral, y comprensión y expresión escrita), claridad de las explicaciones y de los ejercicios de gramática, utilidad de los ejercicios de vocabulario, satisfacción con el contenido técnico relacionado con sus carreras, interés de los temas tratados, variedad y originalidad de las tareas, grado de disminución de la ansiedad al enfrentarse al examen real del FCE (22 ítems).

3. Autoevaluación y comentarios personales: los estilos de aprendizaje preferidos, tipos de recursos externos consultados para realizar los ejercicios, grado de satisfacción al estudiar de forma autónoma, percepción personal de la mejora de sus conocimientos lingüísticos, recomendaciones de mejora, recomendaciones para eliminar o añadir elementos al curso, preferencias de los temas tratados (26 ítems).

4. Sesiones voluntarias de práctica oral: grado de satisfacción con las herramientas utilizadas, calidad de la interacción entre los compañeros de clase, con los tutores nativos y con los profesores (5 ítems).

5. Posibles mejoras: la inclusión de más recursos audiovisuales, de tareas de telecolaboración con estudiantes de otros países, la incorporación de actividades a través de mundos virtuales, etc. ( 5 ítems).

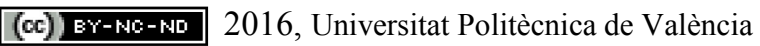


Cada variable estaba compuesta, por tanto, de una serie de elementos definitorios y analizados de forma cualitativa y cuantitativa.

\section{Resultados}

\subsection{Cuestionario inicial}

El cuestionario inicial nos proporcionó una gran cantidad de información sobre el perfil de los estudiantes. Más de la mitad de ellos $(61 \%)$ se veían a sí mismos como buenos estudiantes de inglés aunque el $67 \%$ reconoció que le preocupaba cometer errores y el 75\%, que sentía vergüenza al hablar en inglés en público, de lo que se deduce que existe un alto grado de inseguridad entre los estudiantes a la hora de enfrentarse a una situación de comunicación real en inglés. La vergüenza, por tanto, se perfila como uno de los lastres con los que han de lidiar los estudiantes. Por este motivo, uno de los empeños al diseñar el curso online fue precisamente intentar ayudar a los alumnos a fomentar su autoconfianza, proporcionando consejos y técnicas para afianzar el aprendizaje. Por otra parte, el $84 \%$ de los encuestados afirmaron que, en su opinión, el hecho de tener un buen dominio de inglés es fundamental para su futuro profesional, siendo esta convicción una de sus mayores motivaciones para seguir estudiando la lengua. A esto añadimos que el $95 \%$ de ellos piensa que tener un buen nivel de inglés les ayudará a mejorar sus perspectivas de trabajo en el futuro. Otros motivos alegados que les incita a querer mejorar su nivel de conocimiento son el deseo de viajar (84\%) y un interés por conocer y comprender otras culturas $(86 \%)$.

Algo que resultó sorprendente, sin embargo, fue que contestaron que, aunque la tecnología formaba parte de su vida diaria y que utilizaban Internet en inglés como apoyo para el estudio (por ejemplo, para buscar terminología o información específica, etc.), ninguno de ellos había utilizado nunca un curso online para estudiar inglés. La novedad de utilizar el curso online InGenio FCE Online Course \& Tester y la convicción de que los materiales basados en la tecnología ofrecen mayor variedad, libertad de acceso e incluso entretenimiento, les predispuso muy favorablemente a utilizar nuestro curso para prepararse para el examen del First Certificate aunque esto también aumentó considerablemente sus expectativas respecto a la calidad y efectividad de los materiales. Fue uno de los estudiantes, sin embargo, que al contestar a una de las preguntas abiertas, dio en el clavo. Dijo:

[N44] In my opinion, it's all about the way you present the educational materials and the methods you are applying to learn. The strong point of using technology is the multimedia capabilities that it implies, like integrating text, image, video and sound. But it won't work if the method is boring and the display is not appealing.

Como vemos, este estudiante es consciente de que, por mucha tecnología que se utilice, si no hay una buena metodología subyacente, no es en sí misma ninguna garantía. Naturalmente los materiales didácticos han de estar muy bien planificados y diseñados para que cumplan sus objetivos.

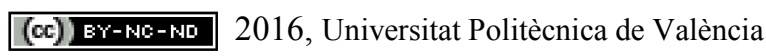

Congreso In-Red (2016) 
Otra ventaja que destacaron reiteradamente respecto al uso de tecnología fue la posibilidad de recibir feedback inmediato tras la realización de un ejercicio en lugar de tener que esperar a que el profesor o profesora se lo corrigiera y le comentara los posibles errores. Cuando se les preguntó si creían que un entorno de aprendizaje virtual imponía menos que una clase presencial, un $45 \%$ afirmó que así era. Esto supone casi la mitad del grupo, lo que nos remite de nuevo a la idea de que lo que en realidad les impone es el hecho de tener que participar activamente en clase, además del temor y la vergüenza de hablar y cometer errores en público. El 83\% reconoció que la expresión oral era su punto más débil. Muy alejados de este porcentaje, hubo un 13\% que reconoció que lo era la comprensión oral, un $4 \%$, la expresión escrita y $0 \%$, la comprensión escrita. Otro hecho interesante es que el $88 \%$ de los estudiantes afirmaron que el medio de instrucción les influía en su motivación de estudiar y la totalidad de ellos apuntó que les resulta interesante estudiar inglés online, lo que significa que su actitud era del todo favorable a estudiar inglés con nuestro curso de InGenio. A la pregunta que formulaba su preferencia por estudiar de forma individual o colaborativa, las opiniones estaban divididas en ambos sentidos. El $60 \%$ dijo preferir trabajar en equipo y el restante $40 \%$, de forma individual, lo que, en principio, puede hacer pensar que este segundo grupo estaría más dispuesto a estudiar una lengua extranjera a través de un curso online, fundamentalmente basado en el autoaprendizaje.

Respecto a cómo prefieren estudiar y practicar la lengua, el $41 \%$ afirmó que preferían un contexto presencial con un profesor en clase, seguido de un $36 \%$ que dijo preferir una clase presencial utilizando tecnología en el aula, un 19\% que prefería un entorno virtual con la ayuda de un tutor al que poder consultar de forma remota, y sólo un 3\% dejó constancia de preferir un curso online de autoaprendizaje sin asistencia alguna. Esto enlaza con la idea de que el 56\% del total afirmó que si pudieran escoger, preferirían un contexto de aprendizaje mixto (blended-learning), probablemente influidos por el deseo de tener lo mejor de ambos mundos.

\subsection{Cuestionario final}

Respecto a las variables que se analizaron en el primer apartado del cuestionario final, pudimos constatar que la gran mayoría de los estudiantes estaban satisfechos con el curso en general. Como las preguntas se basaban en una escala Likert de 7 puntos, sólo se tendrán en cuenta como respuestas favorables aquellas que obtengan entre un 5 (por encima de la media) y un 7 (máxima puntuación). Los resultados que se obtuvieron fueron los siguientes:

En lo que se refiere a la estética general del curso:

- E1 $75 \%$ opinó que la interfaz gráfica era amigable.

- $\quad$ El 79\%, que la navegación por el curso era intuitiva.

- El 77\% de los estudiantes afirmó que alguien sin muchos conocimientos informáticos podría sacarle provecho al curso online.

- A un 77\% de ellos les parecieron adecuados los gráficos y los símbolos.

- Para el 66\% resultaron agradables la gama de colores utilizados, las fuentes y sus tamaños y los iconos, etc.

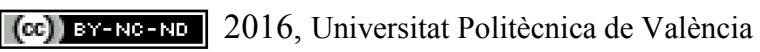


En lo que se refiere a los contenidos del curso:

- El 80\% afirmó que los temas tratados, así como la forma de exponerlos les había resultado interesante.

- Para el $72 \%$, el sonido incorporado era de buena calidad y de un volumen adecuado.

- Un 68\% opinó que las explicaciones grabadas en vídeo (los "Polimedia") eran útiles e interesantes, mientras que las "Course units" obtuvieron un $88 \%$ y las "Tester units", un $86 \%$.

- La cantidad de contenido teórico para dar apoyo al aprendizaje autónomo fue valorado positivamente por un $86 \%$.

- El nivel meta (B2 del MCERL) le resultó satisfactorio a un 90\%.

El tiempo estimado para completar el curso se calculó entre 45 y 60 horas dependiendo de los conocimientos previos de los alumnos. En este sentido, y en relación a cómo interactuaron los estudiantes con el curso online, cabe destacar que el $66 \%$ del total opinó que el tiempo asignado para su realización fue adecuado y suficiente para completar todas las tareas aunque hubo también un $21 \%$ nada despreciable que consideró este tiempo apurado. Sin embargo, si tenemos en cuenta que en las asignaturas de grado en la UPV se estima que el estudiante habrá de dedicarle un 50\% adicional a las horas presenciales de la asignatura, en realidad los estudiantes habrían de dedicarle un total de 112,5 horas al curso InGenio FCE Online Course \& Tester, al sumarle ese 50\% añadido a los 4,5 ECTS de los que consta la asignatura, que asciende a otras 67,5 horas de trabajo autónomo por parte del alumno. Seguramente ese $21 \%$ de alumnos que consideró el tiempo escaso, no era consciente de este hecho.

También resulta interesante el que un 69\% afirmó haber consultado el apartado de teoría de cada unidad didáctica antes de comenzar a realizar los ejercicios, lo que se correlaciona con el hecho de que el $86 \%$ consideró que las explicaciones teóricas eran suficientes y adecuadas, como hemos visto en el listado de arriba. Por el contrario, sólo el $\mathbf{5 8 \%}$ afirmó haber visto los vídeos explicativos (grabaciones "Polimedia"), grabados por los propios autores del curso, antes de iniciar las tareas, lo cual no se ajusta a lo recomendado en las instrucciones de uso. Al preguntarles si opinaban que el curso apoyaba el aprendizaje autónomo, un apabullante $93 \%$ opinó favorablemente. Esto nos hace pensar que han resultado ser efectivas las medidas que se incluyeron para fomentar la independencia de los estudiantes al afrontarse a los contenidos de forma autónoma, aunque -eso sí- contando en todo momento con el apoyo del profesor. Esto nos hace pensar que los estudiantes se han sentido respaldados al poder ir comprobando su progreso tras la realización de las actividades. Una de las razones que, sin duda, ha conducido a esta satisfacción es el hecho de que InGenio incluye un sistema de autoevaluación que permite a los usuarios hacer un constante seguimiento de los resultados obtenidos en los ejercicios. De hecho, el $81 \%$ valoró positivamente el poder acceder en cualquier momento a los informes de evaluación automática que proporciona el sistema, lo cual se correlaciona también con la afirmación del $74 \%$ de que el feedback automático que recibieron fue de utilidad y provechoso para ellos. Además, un $77 \%$ afirmó que disfrutaron de la libertad de poder organizar su propio

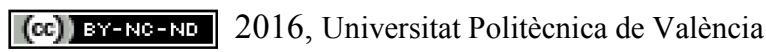

Congreso In-Red (2016) 
tiempo en lugar de estar sujetos a un horario de clase estricto. En línea con estos resultados, el $85 \%$ de los usuarios afirmaron que recomendarían el curso InGenio FCE Online Course $\&$ Tester a un amigo o compañero.

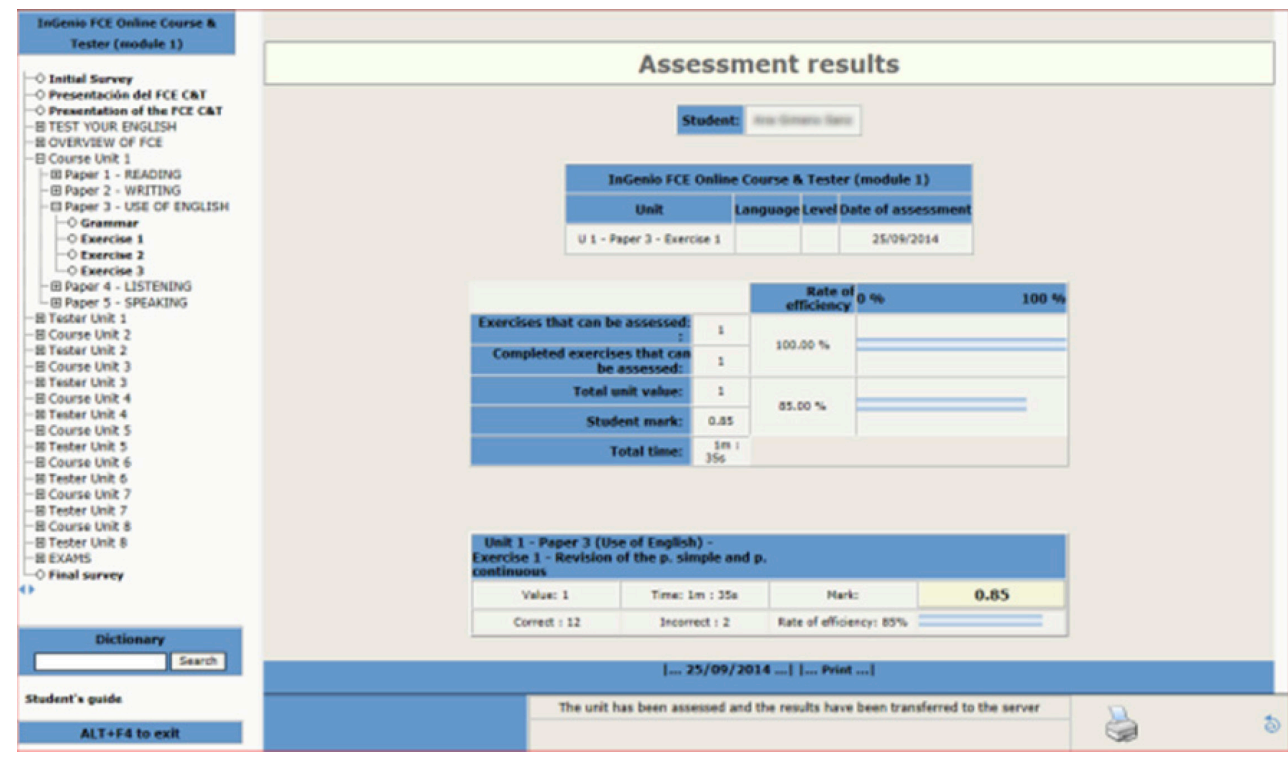

Fig. 2 Ejemplo de informe de evaluación generado automáticamente por el sistema

Dado que al curso se accede a través del gestor de contenidos de la Universidad, PoliformaT, los estudiantes podían comunicarse con su profesor/tutor de forma asíncrona a través del sistema de mensajes de la plataforma o de forma síncrona, a través del chat integrado. Este hecho, sin duda, contribuyó a la satisfacción manifestada por el $89 \%$ de los estudiantes respecto al apoyo recibido por su profesor/tutor. Su percepción en este sentido fue muy favorable.

Los siguientes aspectos sobre los que se consultó se refieren todos ellos a los contenidos del curso. Todos los ítems recibieron un valor que oscilaba entre el $70 \%$ y el $86 \%$ de satisfacción excepto las actividades orales programadas, que obtuvieron únicamente un $\mathbf{5 8 \%}$. Según explicaron los estudiantes en las respuestas abiertas en las que se les pidió que sugirieran mejoras, este valor -inferior a los otros- se debe al modo en que se llevaron a cabo las actividades orales. Un $10 \%$ de ellos aconsejó programar sesiones presenciales grupales con un profesor a lo largo del semestre. Este resultado concuerda con otros estudios (no publicados) llevados a cabo por el Grupo de investigación CAMILLE y por otros investigadores que han podido constatar que los estudiantes se benefician menos de actividades orales "automatizadas" en las que se les pide que respondan a algún input y se graben para que el tutor evalúe su producción oral con posterioridad, comparado con el hecho de recibir feedback por parte del profesor durante una actividad presencial en la que, además, los alumnos están escuchando e interactuando en una situación comunicativa real, aunque se produzca en el contexto de una clase (Zou, 2013, p. 93). Naturalmente, la primera modalidad les priva de una situación comunicativa real y de recibir feedback correctivo inmediato. Sin embargo, todavía hoy en día es más fácil programar

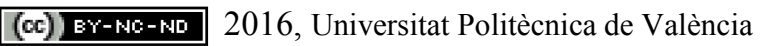


informáticamente actividades para practicar las destrezas pasivas, como la comprensión oral y escrita, que las destrezas activas, como la expresión oral y escrita. Los aspectos con los que los alumnos manifestaron satisfacción incluían: la precisión y claridad de las instrucciones y los encabezados de los ejercicios; utilidad y provecho de los ejercicios de gramática, así como de los de comprensión oral y escrita y expresión escrita; utilidad de las explicaciones teóricas en vídeo describiendo cómo escribir diferentes tipos de textos; adecuación del contenido técnico-científico; adecuación de los ejercicios de vocabulario; interés de los temas tratados y de las actividades asociadas; originalidad, variedad y relevancia de la tipología de ejercicios; utilidad de incluir un "test de nivel" al comienzo para darles a conocer su nivel de partida al empezar con el curso; y utilidad de los consejos y toda la información incluida sobre el examen del First Certificate in English.

La percepción personal de los encuestados cuando se les preguntó si su nivel de inglés había aumentado, dio un porcentaje de satisfacción de un $84 \%$, distribuido de la siguiente forma: extremadamente satisfecho, un $17 \%$; muy satisfecho, un $32 \%$; y satisfecho, un $35 \%$, mientras que el $14 \%$ no tenía opinión y el $3 \%$ manifestó estar un poco insatisfecho. Se obtuvieron unos resultados parecidos (con una satisfacción del 86\%) respecto al aumento de confianza para presentarse y aprobar el examen del FCE, aunque opinaron que el hecho de completar el curso online no contribuía necesariamente a disminuir el nivel de ansiedad que le produciría presentarse a dicho examen. Sólo el $\mathbf{5 6 \%}$ se mostró satisfecho con este aspecto.

En tanto que diseñadores del curso online, acordamos al inicio del proyecto cuestionar y volver a plantear cualquier variable del curso que hubiera recibido un valor de satisfacción por debajo del $60 \%$ (un poco por encima del término medio) para intentar alcanzar la máxima satisfacción posible por parte de los usuarios. Esto implicaba que había tres claros aspectos que requerían un replanteamiento. Tal y cómo se ha mencionado con anterioridad estos aspectos estaban relacionados con a) el visionado de los vídeos teóricos antes de iniciar una tarea; b) las actividades para la práctica oral, y c) aspectos relacionados con la disminución de la ansiedad al afrontar el examen del FCE real. Las soluciones que se pusieron en marcha para resolver estas tres variables fueron las siguientes: a) incluir indicadores más visibles resaltando la conveniencia de ver esos vídeos explicativos; b) programar sesiones presenciales para la práctica oral en grupos de aproximadamente 10 alumnos (12 horas en total); y, c) enfatizar el hecho de que si superaban los ejercicios de la parte del "Tester", que simulan los del propio examen oficial, con límite de tiempo y de intentos, debían confiar en que por la misma razón superarían aquel examen. Como seguimiento de esto, el grupo de investigación tiene previsto realizar un estudio para analizar los resultados de los alumnos que se presentan al examen del First Certificate in English y compararlos con los resultados que obtuvieron en el curso InGenio FCE Online Course \& Tester para ver si éstos se correlacionan. 

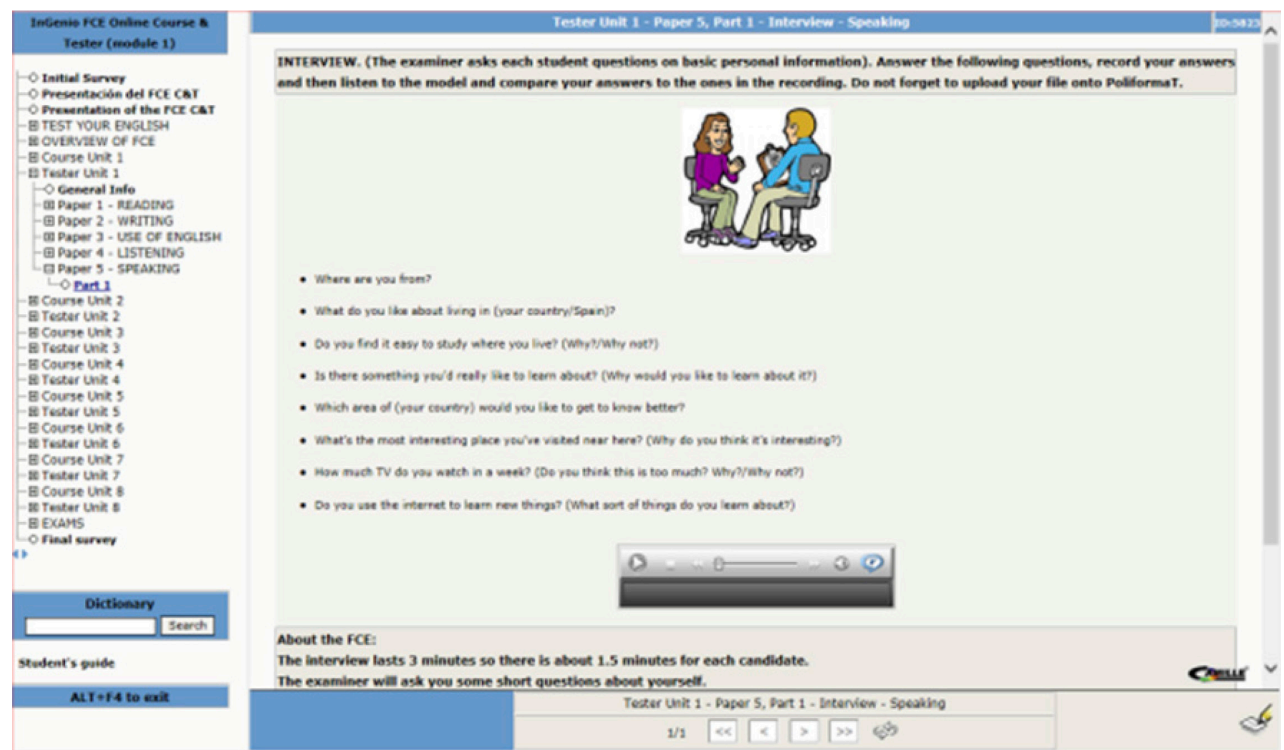

Fig. 3 Ejemplo de actividad oral tomada del Tester Unit 1, paper 5

\section{Conclusiones}

El proceso de validación realizado por medio de un cuestionario inicial y otro final contestados por todos los estudiantes que completaron todos los ejercicios del curso InGenio FCE Online Course \& Tester a lo largo de tres cursos académicos consecutivos proporcionó información muy valiosa para determinar aquellos aspectos que se debían mejorar para incrementar el grado de satisfacción de los estudiantes.

El aspecto que predomina en lo que a mejoras se refiere, es sin duda la práctica oral -es decir, el perfeccionamiento de la expresión oral en contextos de comunicación real. De todas las actividades incluidas en el curso, fue la práctica oral la que menos satisfacción produjo. Desde su punto de vista, las actividades orales no les habían estimulado principalmente por su falta de interactividad, teniendo como consecuencia la disminución de su motivación. Sin embargo, también es cierto que quizás sus expectativas eran mayores de lo que nos puede ofrecer hoy en día la tecnología. Acorde con esto, en el "Study on the impact of information and communications technology (ICT) and new media on language learning" (2007, anexo III, p. 64) encomendado por la Agencia Ejecutiva en el ámbito Educativo, Audiovisual y Cultural de la Comisión Europea se informaba que en opinión de los entrevistados "many learners [are] aware of [the] benefits of ICT/new media for developing reading/writing skills, [but] fewer believe they can develop speaking/listening skills." Además, Zou observó en un estudio publicado en 2013 que el 84\% de los estudiantes encuestados afirmaron que las indicaciones de sus profesores les ayudó a mejor su producción oral mucho más que estudiando en solitario con su ordenador (p. 91). Por ende, creemos que, de momento, la mejor forma de fomentar el inglés hablado con el curso InGenio FCE Online Course \& Tester es, como se ha mencionado anteriormente, organizar 
actividades complementarias con un tutor, bien en sesiones presenciales o a través de vídeo conferencia.

Por último, nos gustaría enfatizar el hecho de que los datos recogidos en ambas encuestas apoya la idea de que los estudiantes se sienten atraídos por la tecnología y creen firmemente en sus ventajas para ayudarles a mejorar su nivel de inglés de forma autónoma y efectiva.

\section{Referencias}

MINISTERIO DE EDUCACIÓN, CULTURA Y DEPORTE (2002). Marco común europeo de referencia para las lenguas: aprendizaje, enseñanza, evaluación. Madrid: Anaya. Disponible en <http://cvc.cervantes.es/ensenanza/biblioteca_ele/marco/cvc_mer.pdf $>$ [Consulta: 15 de marzo de 2015].

STEVENS, A. y SHIELD, L. (2007). Study on the impact of information and communications technology (ICT) and new media on language learning. European Commission. Disponible en $<\mathrm{http} / / /$ eacea.ec.europa.eu/llp/studies/study_impact_ict_new_ media_language_learning_en.php> [Consulta: 24 de septiembre de 2014].

ZOU, B. (2013). Teachers' support in using computers for developing students' listening and speaking skills in pre-sessional English courses. Computer Assisted Language Learning, 26:1, 83-99, DOI: 10.1080/09588221.2011.631143.

\footnotetext{
i El Grupo de Investigación CAMILLE de la UPV tiene más de 25 años de experiencia en el desarrollo de cursos de idiomas multimedia en formato electrónico y ha publicado una decena de cursos para el aprendizaje de lenguas extranjeras a través de internet.

${ }^{i i}$ Los coautores son Antonio Martínez Sáez, Ana Sevilla pavón, Jose Macario de Siqueira Rocha, Roberto

González y Ana Gimeno Sanz.

iii Para más información sobre el sistema InGenio, váese http://camilleweb.upv.es/camille; http://eurocall.webs.upv.es/index.php?m=menu_00\&n=news_07\#projects; Gimeno Sanz, A. (2008). "InGenio: A dedicated CALL authoring shell, content manager and courseware delivery platform". Aprendizaje de lenguas asistido por ordenador: herramientas de autor para el desarrollo de cursos a través de la web. Valencia: Editorial de la Universitat Politècnica de València, pp. 93-117; y de Siqueira Rocha, J.M. (2012). Desarrollo y validación de soluciones tecnológicas para el aprendizaje a través de la plataforma de e-learning InGenio. Valencia: Editorial de la Universitat Politècnica de València. Disponible en

http://www.upv.es/pls/obib/sic_publ.FichPublica?P_ARM=5529.
} 